\title{
The influence of Rosemary plants (Rosmarinus officinalis) volatiles on
}

\section{Aphid (Myzus Persicae)}

\author{
Tingting Cai ${ }^{1, a}$, Liang Wang ${ }^{2, b}$, Kun Zhang ${ }^{3^{*}, \mathrm{c}}$, \\ Zhonglong Lin ${ }^{4,}$, Rong Wang ${ }^{5, \text { e }}$ \\ ${ }^{1}$ Yunnan Forestry Technological College, Kunming, Yunnan, 650224, China \\ ${ }^{2}$ Yunnan Tobacco Company Kunming Branch, Yunnan Kunming 650051, China \\ ${ }^{3}$ Editorial Department of Journal of Southwest Forestry University, Kunming 650224, China \\ ${ }^{4}$ Yunnan Tobacco Company, Yunnan Kunming 650000, China \\ ${ }^{5}$ Yunnan Green Leaf Health Science and Technology Co., Ltd, Yunnan Kunming 650051, China \\ ${ }^{a}$ ctingting2004@163.com, ${ }^{\mathrm{b}}$ 4406815369@163.com, \\ c*53508559@qq.com, dsdlzl1983@163.com, ${ }^{\mathrm{e}}$ 1036254195@qq.com
}

Keywords: behavioral responses, rosemary, Myzus persicae, Y-tube olfactometer.

Abstract: Volatile organic compounds (VOCs) can have an important influence on plant-insect interactions. In particular, insect behaviors are often influenced by the volatiles of both host plants and non-host plants. The objective of this study was to assess the behavioral responses of the aphid, Myzus persicae (Sulzer) to the volatiles of the woody perennial herb Rosemary (Rosmarinus officinalis, family Lamiaceae). Our studies using a range doses of plants have shown that, at all of the different doses tested except the weight of rosemary was $0.5 \mathrm{~g}$, volatiles emitted from rosemary plants showed repellent effects on $M$. persicae. These findings provide new insights into the interactions between $R$. officinalis plants and $M$. persicae, which will contribute to the development of new management strategies for biological control of insect pests

\section{Introduction}

Plants naturally not only literally building forests from gases taken from the air but also releasing complex bouquets of volatile organic compounds (VOCs) back into the air, These chemical signatures can be used by predators to locate their prey or hosts ${ }^{[1]}$.Thus, VOCs can have an important influence on plant-insect interactions. For example, host plant volatiles can regulate insect behaviors ${ }^{[2,3]}$. The volatiles of maize (Zea mays L.) are attractive to Western corn rootworm adults (Diabrotica virgifera) ${ }^{[4]}$, and the odour of oilseed rape (Brassica napus) attracts cabbage seed weevils (Ceutorhynchus assimilis) ${ }^{[5]}$. Moreover, non-host plants volatiles also can influence insect behaviors ${ }^{[6]}$; Cymbopogon citrates, a plant with known repellent activity, has a repellent effect against mosquitos ${ }^{[7]}$, as do volatiles of the neem tree ${ }^{[8,9]}$. Thus, intercropping of host plants with non-host plants can be used, so that the VOCs of non-host plants disrupt the ability of pest insects to find host plants. This may be an effective means of biological control of insect pests.

The aphid, Myzus persicae (Sulzer), is one of the main pests that feed on the leaves of peach (Prunus persica) trees, cabbage (Brassica oleracea L.) or tobacco (Nicotina tabacum) and other 
plants, to the extent that its aggregative behaviors can seriously reduce the yield and quality of peaches or tobacco or other vegetables. Although chemical control is effective, overreliance on this method carries health and environmental risks associated with the residues that remain on plant leaves, along with the resistance that herbivores may develop, which in turn may lead to the resurgence of primary pests and outbreaks of secondary pests ${ }^{[10]}$. Therefore, it is prudent to explore alternatives to chemical control to improve the sustainability of pest management ${ }^{[11]}$. Methods that alter herbivore behavior or manipulate populations of natural enemies have been recognized as potential alternative tools for the control of insect pest populations ${ }^{[12]}$.

Field observations have indicated that rosemary (Rosmarinus officinalis, Lamiaceae), a natural aromatic crop that originated in the Mediterranean, is never damaged by aphids. We hypothesized that the volatile compounds present in rosemary may have repellent effects to vegetable aphids. Some studies have shown that rosemary essential oil has insecticidal properties ${ }^{[13,14]}$. Isman et al. ${ }^{[13]}$ studied the insecticidal effects of rosemary oils on larvae of the moths Pseudaletia unipuncta and Trichoplusia $n i$ in relation to the oils' chemical compositions. Hori et al. ${ }^{[14]}$ found that rosemary oil as well as thyme oil strongly repelled the aphid Myzus persicae (Sulzer), and rosemary oil has been shown to repel other aphid species such as Aphis gossypii Glover, Macrosiphum euphorbiae ${ }^{[15]}$, and Neotox-optera formosana ${ }^{[16,17]}$. However, the role of rosemary volatiles in mediating interactions between rosemary plants and aphids that feed on crops, has not been investigated. The main objective of this study was to test whether rosemary volatiles have repellent effects on aphids that feed on crops. Confirmation of such properties would provide a useful basis for the development of intercropping methods with rosemary, in order to reduce damage caused by aphids to peach trees and other plants.

\section{Materials and Methods}

\section{Plants}

Rosemary plants were reared in plastic pots $(11 \mathrm{~cm}$ diameter, $9 \mathrm{~cm}$ height $)$ in a climate-controlled greenhouse room $\left(25^{\circ} \mathrm{C}, 70 \% \mathrm{RH}, \mathrm{L} 16\right.$ : D8) and watered every three days in the morning. No fertilizers were applied.

\section{Insects}

The ablate adult aphids ( $M$. persicae) were originally collected from infested tobacco, at the Yunnan Agricultural University, Kunming, China. They were all reared on potted tobacco (at the stage with 5-12 true leaves) in well ventilated plastic cages $(50 \times 50 \times 50 \mathrm{~cm} 3) \mathrm{kept}$ in separate greenhouse chambers at $22 \pm 2{ }^{\circ} \mathrm{C}, 50 \%-72 \% \mathrm{RH}$, and a photoperiod of 16:8 L:D.

\section{Y-tube Olfactometer Bioassay}

Behavioral responses of the aphids to rosemary volatiles were investigated with a glass Y-tube olfactometer ( $1 \mathrm{~cm}$ in diameter, $10 \mathrm{~cm}$ stem and $10 \mathrm{~cm}$ arms, set at a $75^{\circ}$ inner angle). Air was pumped through an activated charcoal filter to remove any contaminating odors and a humidifier was used to humidify the air, divided by a glass Y-junction. The two airflows then passed through two separate flow meters, which regulated the flow rate to $133 \mathrm{~mL} / \mathrm{min}$. The air passed into two glass cylinders $\left(11 \times 19 \mathrm{~cm}^{2}\right)$ with quick fit lids, into which the volatile sources were placed. From there, the air from the glass cylinder flowed into the arms of the olfactometer. All tests were conducted in a climate room $\left(25 \pm 1^{\circ} \mathrm{C}, 50-70 \% \mathrm{RH}\right)$ under a 55-W fluorescent lamp that emitted daylight wavelengths, fitted with a prismatic filter to ensure a completely even distribution of light. This illuminated the Y-tube with a light intensity of 865 lx.

For each of the bioassays, 50 adult aphids were tested following established protocols ${ }^{[18]}$. The aphids were introduced individually into the entry arm. Timing started when the individual reached 
the beginning line ( $3 \mathrm{~cm}$ from the stem inlet), and we then began to observe their choices in the Y-tube Olfactometer. The observation period ended when the individual reached the finish line (7 $\mathrm{cm}$ from the Y-junction) of one of the olfactometer arms, with a maximum observation duration of 5 min per test. Individuals that did not choose a side within 5 min were recorded as "no choice". After five insects had been tested, the Y-tube was cleaned with alcohol (75\%) and distilled water, left to dry, and subsequently the odor sources were switched between the left and right side arms to minimize any spatial effect on choices. Each individual was tested only once. Ten different aphids were used for each replication and five replications were carried out for each treatment.

The experiment was conducted, to examine the response of $M$. persicae to odors emitted from rosemary volatiles versus clean air. Five different masses of aerial parts of rosemary plants were established as five treatments respectively, with five replicates were carried out for each treatment. About 1 hour after taking the five different masses of aerial parts, we placed the aerial parts of rosemary in the glass cylinder respectively, to minimize any mechanical damage to the odor sources.

\section{Statistical Analysis}

For the bioassays with Y-tube olfactometer, Chi-square tests were used to test the hypothesis that the distribution of side-arm choices between pairs of volatiles deviated from the null model that volatile sources were chosen with equal frequency. Independent-sample t-tests were used to assess significant differences between the mean time that was required for individual aphids to make a choice between different volatiles. All the data were analyzed with statistical package SPSS 17.0.

\section{Results}

\section{Response of ablate adult aphids to volatiles of Rosemary}

The results from the olfactometer experiment that investigated insect responses to rosemary volatiles versus clean air showed that aphids were significantly repelled by odors emitted from rosemary at different doses $(p<0.05$ ), (above set) except when the weight of rosemary was 0.5 g. $M$. persicae did not exhibit significantly preference between volatiles from $0.5 \mathrm{~g}$ rosemary and clean air $\left(\chi^{2}=2.00, p=0.157>0.05\right)$ (Fig.1).

There was no significant difference in choice time for the odour sources (i.e., rosemary versus blank treatment), except when the mass of rosemary was $4.0 \mathrm{~g}$ (above set doses) $(p<0.05)$ (Fig. 2 ). When the pair of odors included $4.0 \mathrm{~g}$ of rosemary versus the blank treatment, aphids speed of movement toward the blank treatment was significantly faster than toward the odors of the rosemary $(t=2.21, p=0.032<0.05)$ (Fig.2).

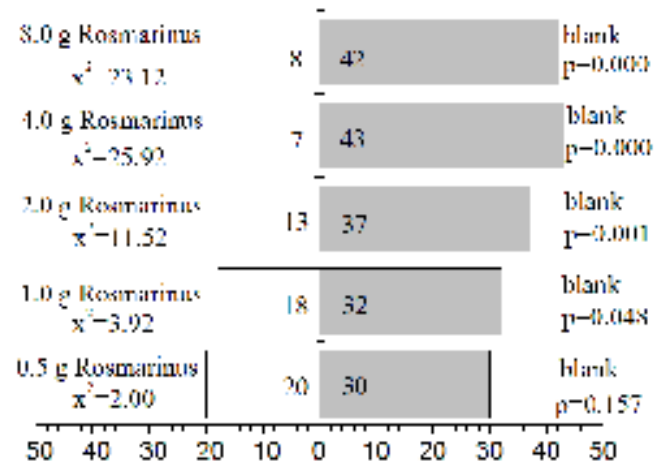

Fig. 1. Olfactory response of $M$. persicae to odors from rosemary versus blank, "Blank" indicates clean air as the control group. Numbers in bars represent individual aphids that moved toward the volatiles on five different experimental doses. 


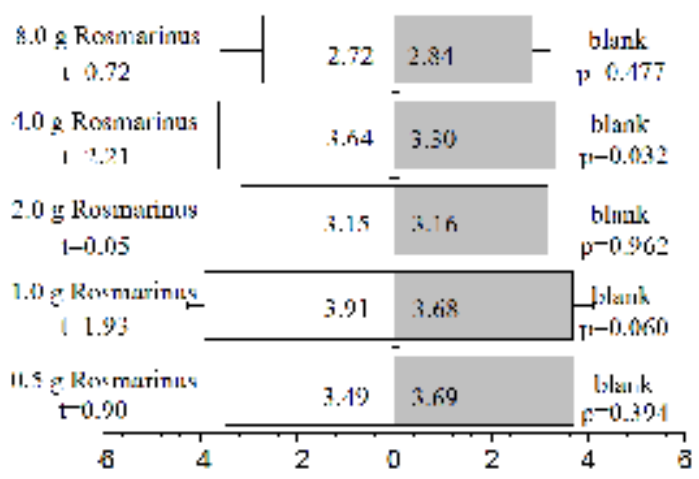

Fig. 2. Olfactory response of $M$. persicae to odors from rosemary versus blank, "Blank" indicates clean air as the control group. Numbers in bars represent the mean time (min) that it took individual aphids to move toward the volatiles.

\section{Discussion}

It is well documented that insect behavior is guided by plant physical cues, such as color and shape, along with short-range chemical cues, such volatile organic compounds of plants, or some secretions produced by insects ${ }^{[19-21]}$. Our study indicates that the behavior of $M$. persicae was influenced by the volatiles of rosemary. M. persicae was repelled by all five doses of rosemary volatiles. These findings suggest that the rosemary volatiles represent a chemical cue that influences the behavior of $M$. persicae, although rosemary is not their host plant. These volatiles may contain some compounds that repel aphids that feed on peach or tobacco or other plants leaves.

In addition, in another olfactometer study, the odour of volatiles from the host plant cabbage attracted $M$. persicae ${ }^{[6]}$, whereas our study indicates that the odour of rosemary volatiles was significantly repellent to $M$. persicae. So, cabbage or peach trees have potential for use in intercropping with rosemary plants, so that the volatiles of rosemary plants may disrupt host finding by aphids. In this way, it may be possible to use non-host plants volatiles to mediate the location of aphids. If successful in the field, such a system could be valuable for reducing the need for chemical applications, and ultimately result in economic benefits to farmers.

\section{Acknowledgements}

This work was financially supported by the College Scientific Research Foundation (KY (BS) 201701) from Yunnan Forestry Technological College and the Guidance Project of Science Fund from Education Department of Yunnan Province (Grant No.2016ZDX176) and the Project of Yunnan Tobacco Company of Science and Technology( 2014YN03).

T.T. Cai, M. Ye, Z. Li, et al.: Advanced Materials Research. 781-784. (2013): p. 737-740.

\section{References}

[1] S.B. Vinson: Annual Review of Entomology. 21 (1976): p. 109-133.

[2] J. Visser: Annual Review of Entomology. 31 (1986): p. 121-144.

[3] D.J. Wei: Acta Photophy Siologica Sinica, 27 (2001): p. 193-200.

[4] B. Hibbard, T. Randolph, E. Bernklau, et al: Environmental Entomology. 26 (1997): p. 285-296.

[5] E. Bartle, M. Blight, A. Hick, et al: Entomologia Experimentalis Et Applicata. 68 (1993): p. 295-302. 
[6] S.F. Nottingham, J. Hardie, G.W. Dawson, et al: Journal of Chemical Ecology. 17 (1991): p. 1231-1242.

[7] W.S. Leal and K. Uchida: Journal of Asia-Pacific Entomology. 1 (1998): p. 217-221.

[8] M.F. Balandrin, S.M. Lee and J.A. Klocke, et al: Journal of Agricultural and Food Chemistry. 36 (1988): p. 1048-1054.

[9] T.T. Cai, X.Q. Lin, P.X. Zhang, et al: Advances in Engineering Research. 115 (2017):p. 312-318

[10] A. Hart, C.D. Brown, K.A. Lewis, et al: Agronomies. 23 (2003): p. 75-84.

[11] L.L. Cui, J. Dong, F. Francis, et al: Crop Protection. 35 (2012): p. 91-96.

[12]L.C. Rodriguez and H.M. Niemeyer: Crop Protection. 24 (2005): p. 615-623.

[13] M.B. Isman, J.A. Wilson, R. Bradbury, et al: Pharmaceutical Biology. 46 (2008): p.82-87.

[14] M.Hori: Journal of Chemical Ecology. 24 (1998): p.1425-1432.

[15] T.I. Ofuya: Crop Protection. 14 (1995): p.47-50.

[16] M. Hori and H. Komatsu: Applied Entomology and Zoology. 32(1997): p.303-310.

[17] J. Takabayashi, S. Takahashi, M. Dicke, et al: Journal of Chemical Ecology. 21(1995): p.273-287.

[18] Y.G. Lou, B. Ma, J.A. Cheng, et al: Journal of Chemical Ecology. 31 (2005): p. 2357-2372.

[19] E. Hatano, G. Kunert, J. Michaud, et al: European Journal of Entomology. 105 (2008): p. 797-806.

[20] J. Michaud and M. Mackauer: Entomologia Experimentalis Et Applicata.70 (1994): p. 273-283.

[21] T.T. Cai, M. Ye, Z. Li, et al.: Advanced Materials Research. 781-784. (2013): p. 737-740. 